\title{
Design And Development of A Plug-In Type Wireless Data Logger for Energy Measurement
}

\author{
D. D. Vyas ${ }^{1}$, H. N. Pandya ${ }^{2}$ \\ ${ }^{I}$ Department of Electronics \& Communication, Darshan Institute of Engg. \& Technology, India \\ ${ }^{2}$ Department of Electronics, Saurashtra University, India
}

\begin{abstract}
Increasing demand of electrical energy and its rising prices have enforced the need of managing usage of electrical energy more efficiently. This requires regular monitoring of electrical appliances to timely identify inefficient energy consumption due to faulty conditions, inefficient designs, user negligence, etc. In this paper, design and development of a low-cost wireless data logger is presented that aims to accurately measure the value of energy and related parameters like $V_{R M S}, I_{R M S}$ and instantaneous power in real time for single phase applications. The proposed data logger acts as a plug-in device that can easily be introduced between mains and the appliance. The system can collect the data in real time and communicate it to a computer over a Bluetooth based wireless link. The computer hosts a simple graphical user interface (GUI) based application that allows user to log the data as and when required. The proposed data logger is designed using CS5460A single phase bi-directional power/energy IC from Cirrus logic interfaced with AVR ATmega32 microcontroller. A wireless link between is established using AUBTM-20 Bluetooth modules. Serial EEPROM is also included in the proposed design to store the data locally on the board for later use. GUI for the data logger has been designed using MATLAB.
\end{abstract}

Keywords - Bluetooth, Data logger, Energy monitoring, , Embedded system, Real time monitoring

\section{INTRODUCTION}

Home as well as industries consumes much more electric energy than required mainly due to inefficient designs, faulty devices and user's negligence. Studies show that about five to fifteen per cent of energy can be saved if devices are monitored and handled efficiently [1]. However, the normal energy metering system that generally exists only give the total amount of energy consumed and thus user is not able to monitor the amount of electricity being used by individual appliance and are also not able to timely identify any drastic difference in power consumption due to faulty condition in the appliance. This proposes a need of a low-cost system that can be easily interfaced with any electric appliance and helps to measure the consumption of electric energy in real time.

In this paper design and development of a plug-in type data logger is discussed that is capable of accurately measuring energy and related parameters like $V_{\text {RMS }}$, $I_{\text {RMS }}$ and instantaneous power in real time for single phase applications. The data logger is equipped with a Bluetooth based wireless communication module that is capable of neatly communicating the sampled data to a computer without any wired interface. Thus the design becomes a plug and play type wireless energy meter that is very simple and flexible to use. Researchers and industries have developed such real time energy monitoring systems, however these requires additional setup and are little less economic [2-5]. The proposed design aims to serve two purposes. The first is to provide a low-cost solution for real time data logging of energy either for testing or continuous monitoring and the second is to encourage consumers for use of such devices enabling them to play a proactive role in efforts towards energy conservation and other goals of smart grid [6-8].

The rest of the paper is organized as follows. In section 2 design requirements and proposed system architecture is discussed. In section 3 and 4 details of hardware and software involved in the design of the system are discussed at length. This is followed by discussion on experimental results in section 5 , future direction of work in section 6 and conclusion in section 7 .

\section{DESIGN REQUIREMENTS AND SYSTEM ARCHITECTURE}

Following design requirements were proposed to be met by the system.

- Compatible to any single phase application connected to standard 16A source.

- Able to monitor energy in real time and the data should be logged into local memory as well as computer if interfaced.

- Wireless data communication with computer avoiding the use of additional cables.

- Able to monitor other parameters like $\mathrm{V}_{\mathrm{RMS}}, \mathrm{I}_{\mathrm{RMS}}$ and instantaneous power in real time when connected to a computer.

- Support of a simple GUI based application on computer for easy user access. 
- Low cost and simple to add and remove.

To meet the mentioned requirements the proposed system architecture is shown in Fig. 1. System on the input side is connected to mains through a standard 16A plug-socket interface and a similar plug-socket interface is available on the output side for connecting the appliance whose energy monitoring is intended. The system is microcontroller based unit with energy measuring module, EEPROM, wireless module for communication and other I/O interfacing modules. Energy measuring module performs the task of measuring energy and related parameters continuously. Microcontroller is supposed to fetch this data at regular interval, update the data in local memory and also forwarded it to computer over a wireless communication link.

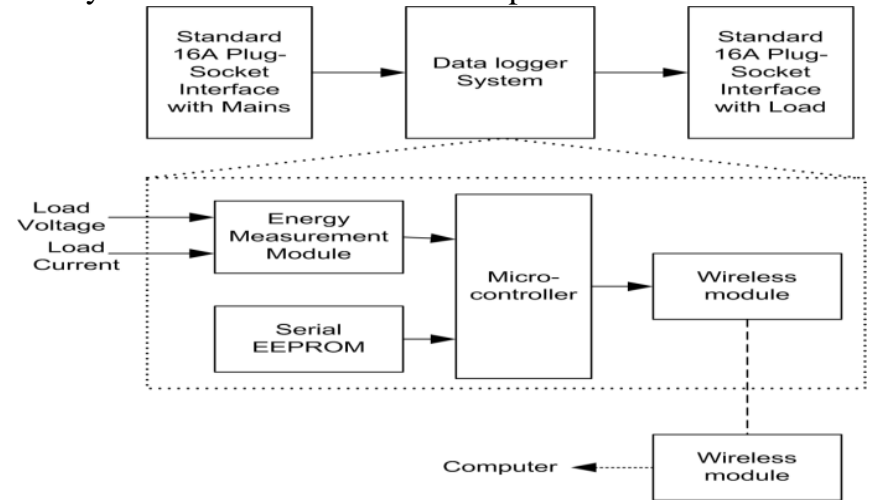

Fig. 1 System architecture

\section{SYSTEM HARDWARE}

System hardware consists of two modules: one for data measurement and transmission and another for data reception and logging on a computer. This is shown in Fig 2(a) and 2(b) respectively. The first module, more complicated of the two, can be partitioned into two main sections: analog and digital as shown in Fig. 2(a).

Analog section in the transmitter module, responsible for energy measurement, is designed using CS5460A. The CS5460A consist of two 24 bit $\Delta \Sigma$ ADCs, high speed power calculation functions with digital filters for signal conditioning and a serial interface all on a single chip. It is designed to accurately measure and calculate: energy, VRMS, IRMS and instantaneous power for single phase 2 or 3 -wire applications. It meets accuracy specification for IEC 687/1036, JIS [9]. It can be interfaced using a simple resistive divider or potential transformer to measure voltage and with low-cost shunt resistor or current transformer to measure current. It supports auto-boot feature to function as stand-alone device as well as a standard bi-directional three wire serial interface (compatible to SPI and Microwire) for communication with microcontroller. The CS5460A also supports on-chip facility for AC or DC system-level calibration for both voltage and current channel. It has internally around twenty 24-bit registers related with configuration, calibration, status and data that can be read or written by microcontroller. A board designed for this analog section is shown in Fig. 3(a).

Digital sections consist of AVR ATmega32 microcontroller, AUBTM-20 based Bluetooth module, serial EEPROM 24c04, LCD and few switches. In addition to the general advantages that ATmega series microcontroller has, the reasons for selecting ATmega32 was that it supports all the three type of serial interface required in this design [10]. These are three wire serial interface required in interfacing with CS5460A, two wire serial interface required in interfacing of serial EEPROM and standard USART for RS232 interface required for

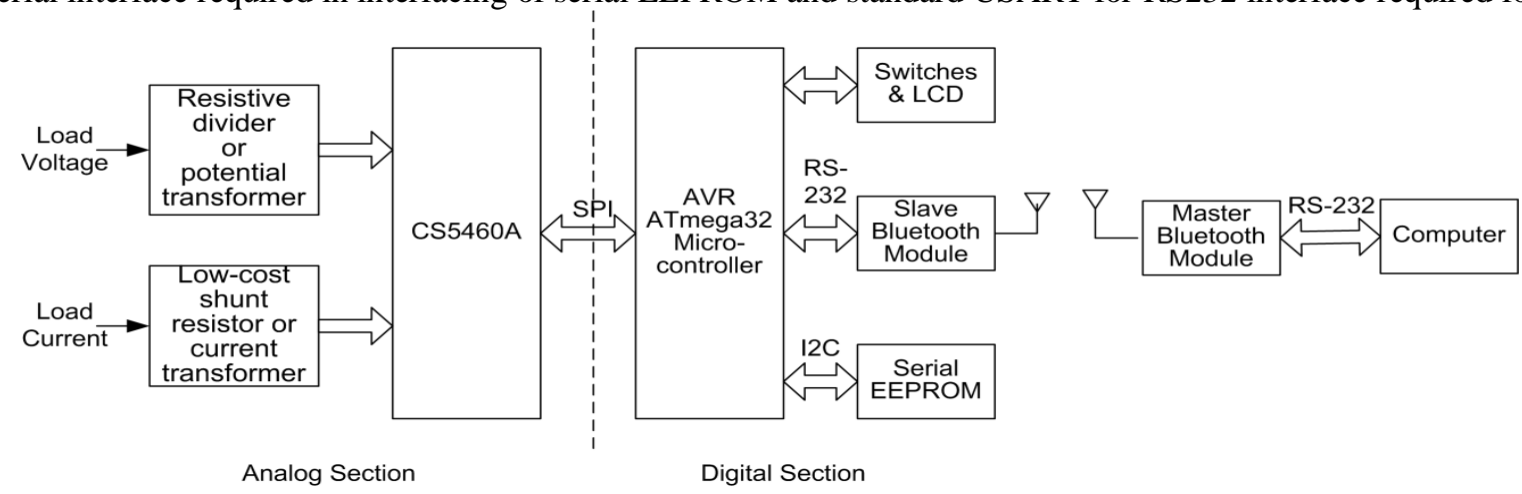

(a)

(b)

Fig. 2. System hardware (a) Measurement and transmission module (b) Receiver module 


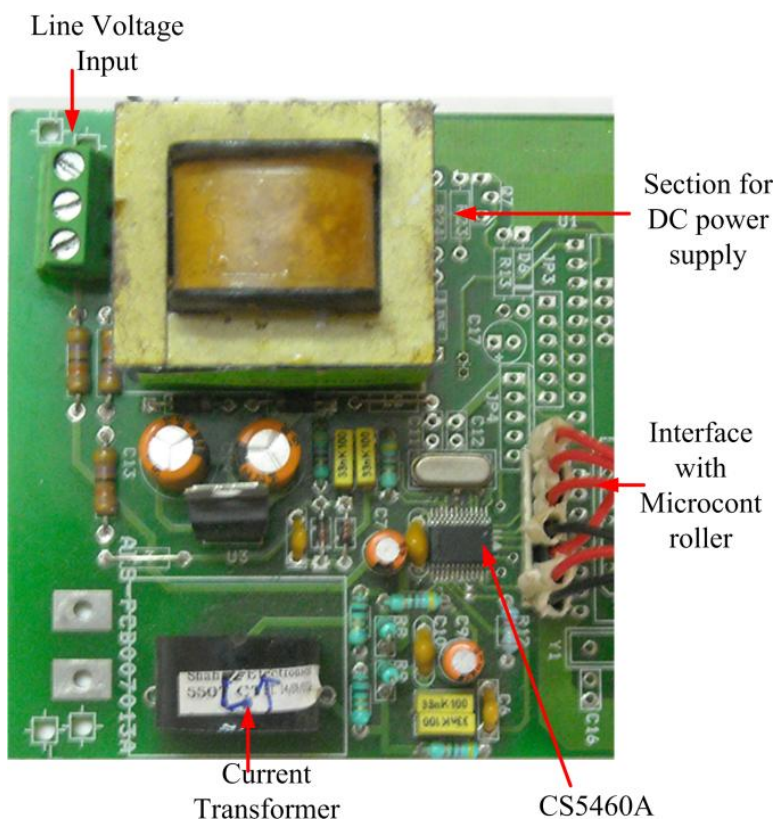

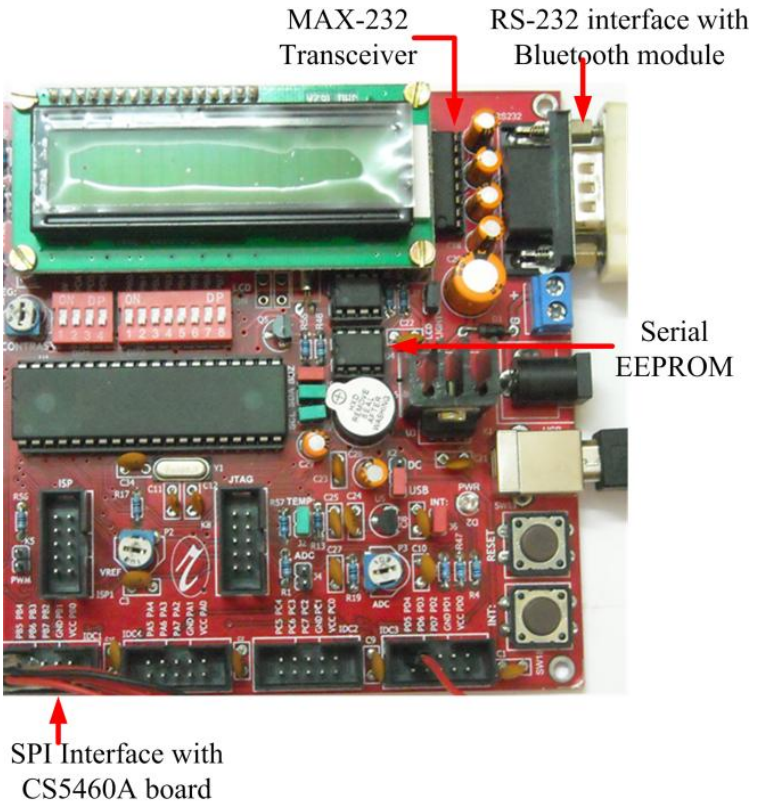

(b)

(a)

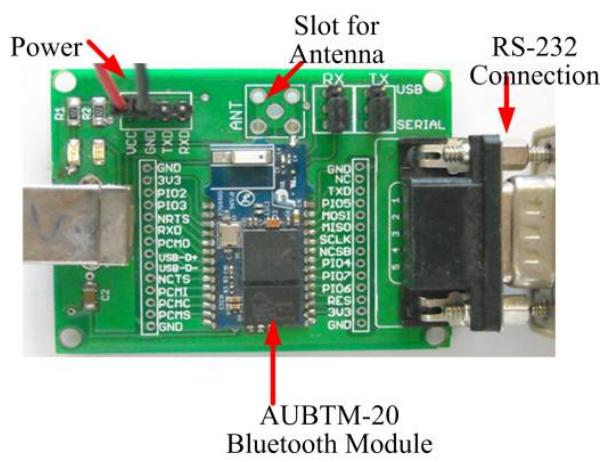

(c)

Fig. 3 (a) CS5460A based energy measurement module (b) ATmega32 based microcontroller module (c)

AUBTM-20 based Bluetooth module

Bluetooth module. Bluetooth module connected with the system is configured as a slave and a similar one connected with a computer, as shown in Fig. 2(b), is configured as a master. When connection is established by the master, the slave will pass the measured data to the master. Once master and slave are connected, to disconnect the link it is required to reset the slave module. This is achieved in the design by use of a simple hardware that enables power to Bluetooth module under the control of ATmega32 microcontroller. This is discussed further in the next section of the paper. The role of on-board serial EEPROM is to store the total energy consumed over the time since the beginning of the measurement. Switches and LCD are provided mainly for debugging and on board monitoring. Board representing the digital section is shown in Fig. 3(b) with Bluetooth module in Fig 3(c).

\section{SYSTEM SOFTWARE}

Systems software consists of two main programs: microcontroller program and GUI application program on computer.

Program on microcontroller consist of two modules, one for initial calibration of CS5460A and second for real time measurements and data logging. Before using CS5460A for measurements for the first time, it has to be calibrated following a specific sequence of operations and these calibrations values are then saved in serial EEPROM for later use during measurement. For both voltage and current channel there are calibration sequence both for AC and DC purpose. Calibration in AC or DC is primarily for two basic types system offset and system gain. Depending on the specific metering application and accuracy requirements, some or all of the calibration sequences may be required to be executed. There are dedicated registers in CS5460A for specific calibration sequence. The steps for calibration process are shown in Fig. 4 (a) with the recommended sequence in Table 1. Calibration of each type requires applying appropriate inputs on the input channels, issuing calibration 
commands and reading result from the concerned registers at the end of calibration. As shown in Table 1, if both AC and DC calibration has to be performed then DC calibration should precede AC calibration and if both offset and gain calibration has to be performed then offset should precede gain [11].

Table 1. Calibration Sequence

\begin{tabular}{lll}
\hline \hline $\begin{array}{l}\text { Preferred } \\
\text { Sequence }\end{array}$ & CALIBRATION TYPE & Inputs applied to V/ I channels \\
\hline 1 & DC offset & Connected to ground level \\
2 & AC offset & Connected to ground level \\
3 & DC gain & DC signal equivalent to absolute peak full \\
& scale value of input \\
4 & AC gain & AC signal with maximum possible RMS \\
& & value of input \\
& \\
\hline \hline
\end{tabular}

Flow chart highlighting the sequence of major operations performed in microcontroller program for real time measurement of energy is shown in Fig. 4(b). On power-on CS5460A is required to be initialized. This involves two operations. First operation is to fetch calibration values that are stored in serial EEPROM and load them into concerned registers in CS5460A. Second operation is to define conversion cycle and computation cycle rate. Conversion cycle indicates the rate at which instantaneous voltage, current and power is obtained and computation cycle indicates number of these instantaneous values that are used to calculate VRMS, IRMS and energy over the period. For example, if conversions cycle is $4000 \mathrm{~Hz}$ and computation cycle is set to one second, then VRMS and IRMS will be calculated using 4000 samples of instantaneous values of voltage and current respectively and energy over one second will be calculated summing 4000 samples of instantaneous power. These cycles are again defined by loading appropriate values in concerned registers. These values depend on the clock frequency of CS5460A.

After initialization of CS5460A the slave Bluetooth module that is connected with microcontroller is enabled i.e. powered ON. This is done by a simple arrangement where a microcontroller enables a buffer that is responsible to provide to the slave module. The reason for this arrangement is that once master and slave modules are connected for data transfer both enter into data mode then to disconnect them and bring them back in command mode when required, one of the module has to be reset.

Further, before interfacing Bluetooth modules in the system they have to be configured for baud rate, mode of operation (master/slave), identification number, etc. This is done by sending series of AT command to Bluetooth module over a RS-232 serial link. Various AT commands used for configuration as well as data transfer are discussed in Table $2[12]$.

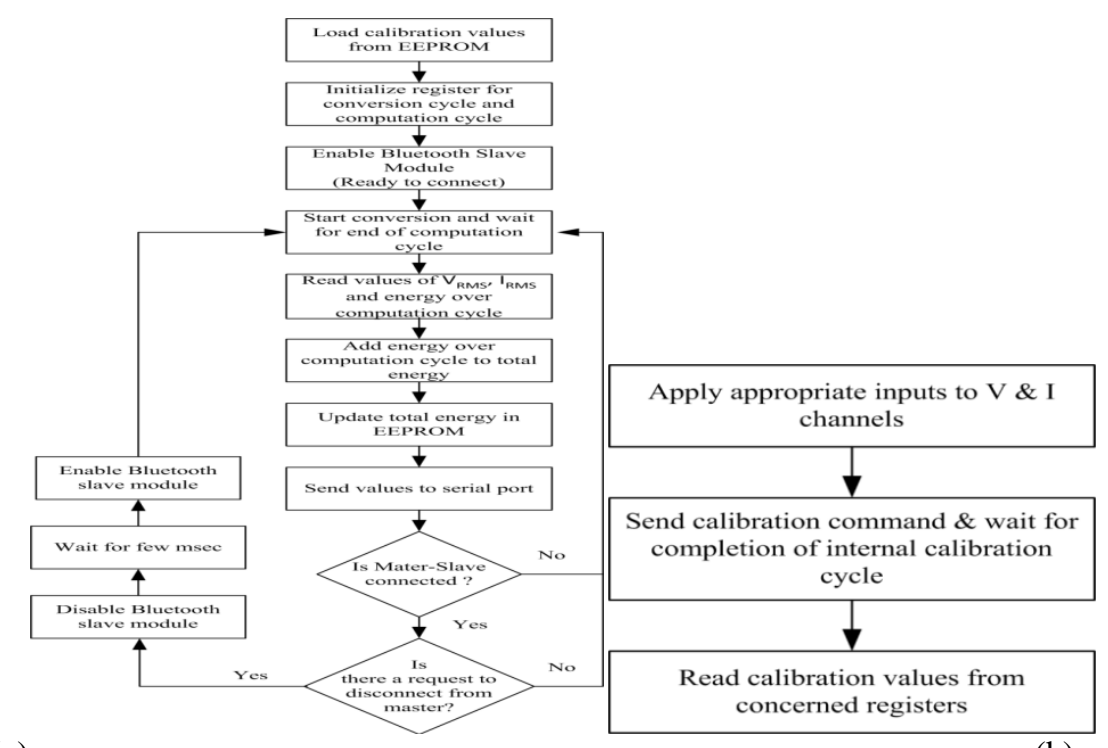

(a)

Fig. 4 (a) Fig. 4 Steps in calibration process (b) Process of acquiring measurements from CS5460A.

After initialization of CS5460A and enabling of Bluetooth module, the process of data measurement starts. Microcontroller handles this process in a polling mode. At end of each computation cycle value of $\mathrm{V}_{\mathrm{RMS}}$, $\mathrm{I}_{\mathrm{RMS}}$ and energy are read. Energy over the period of each computation cycle (e.g. duration of 1 sec.) is accumulated to have the total amount of energy consumed and this count is updated in serial EEPROM. Further 
all the values measured over the computation cycle are by default sent to the serial port where the slave Bluetooth module is connected. Thus when master and slave Bluetooth link is active the measured data is transferred to computer.

Table 2. AUBTM-20 Bluetooth Module AT Commands

\begin{tabular}{ll}
\hline \multicolumn{1}{c}{ AT COMMANDS } & \multicolumn{1}{c}{ Description } \\
\hline AT+SETUP & $\begin{array}{l}\text { Shows current setting of module like baud rate, mode of } \\
\text { operation, address etc. } \\
\text { Define the mode of operation that can be Master, Slave or Auto } \\
\text { Connect. }\end{array}$ \\
AT+MODE & $\begin{array}{l}\text { Set PIN code for the Bluetooth module } \\
\text { Set current active profile that can be either serial port, head set } \\
\text { AT+PIN }\end{array}$ \\
or hands free \\
Discover other Bluetooth modules in the neighborhood. \\
AT+CON
\end{tabular}

GUI based application is developed in MATLAB for data logging on a computer. A snapshot of GUI is shown in Fig. 6. GUI allows user to select slave module by its address (required in case if there are more than one Bluetooth modules around), and specify the duration for which data logging has to be done. On activation GUI application commands master to connect with slave and start logging the value of $V_{\text {RMS }}$, $I_{\text {RMS }}$ and energy that are received after each computation cycle. This continues for the specified duration after which GUI sends signal to slave requesting to disconnect. Measurements received by GUI are stored in from a matrix and remains available for off-line analysis also.

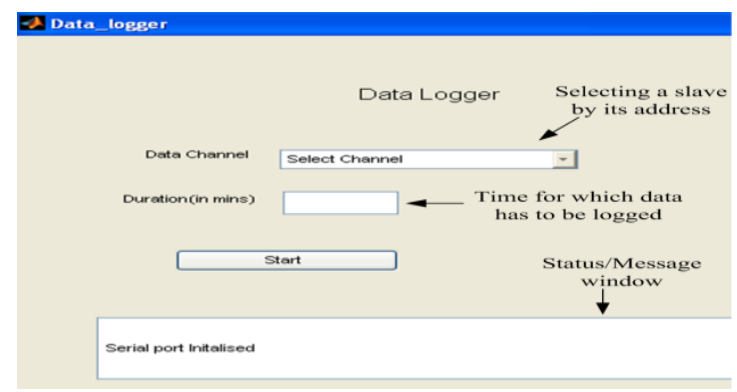

Fig. 6 Snapshot of GUI application for data logging on computer.

\section{EXPERIMENTAL RESULTS}

Designed data logger has been tested for varieties of load of different capacities and type. For example a fixed resistive load, variable resistive load, induction motor etc. Appropriate scaling factors were identified for ADC. This is required because in CS5460A measured value of $V_{R M S}$ and $I_{R M S}$ are available as 24 bit unsigned value in the range of $0.0 \leq V_{R M S}, I_{R M S} \leq 1.0$, whereas that of energy is available as 24 bit singed value in the range $-1.0 \leq \mathrm{E}<1.0$. Further AUBTM-20 is a Version 1.2, class-2 Bluetooth module that should give connectivity range of around 10 meters; however range of successful communication without antenna was found to be less than 2 meters and with antenna was around 8 meters. Maximum bit rate supported by Bluetooth Version 1.2 is around 1 Mbits/s [13]; however as the required bit rate in our case was well below the maximum possible, this was not much of the concern.

The measurements obtained from the data logger were compared with those obtained from parallel connected KRYKARD portable load manager ALM32 from Manaco Energy Solutions Pvt. Ltd. The results were encouraging and very much within the acceptable limits of accuracy.

\section{FUTURE DIRECTIONS}

Direction of the work that is intended to be followed as an ongoing part of the presented research work includes following

- To review alternate technology for wireless link as Bluetooth technology has limitation in terms of communication range and number of nodes. One of the possible alternatives to be studied is Zigbee.

- To have a real time data logging from more than one channel. Here number of data loggers will be used to measure energy of different appliances and computer in turn would read the value from each of the channel.

- To develop a complete home energy management system that can be the part of the smart grid. 


\section{CONCLUSION}

Design and development of a plug-in type wireless data logger for measurement of energy and related parameters for single phase applications has been discussed. The design has an advantage of being easy to use and low cost. GUI has been developed for data logging on a computer and on-line/off-line analysis of data. Operation of the proposed data logger has been verified experimentally and has been found to be meeting acceptable limits of accuracy. It is believed that availability of such low cost easy to use energy monitoring solutions in the market will encourage consumers to use them. This will help them to manage their energy usage more efficiently and also to contribute towards goals of smart grid like efficient energy management and energy conservation. The presented work was part of the ongoing research towards design and development of a fullfledged low cost home energy management system.

\section{REFERENCES}

[1] S. M. Ahluwalia, Power sector reforms: a review of the process and an evaluation of the outcome, NCAER, Delhi, March 2000.

[2] Energy monitoring technologies inc., 2011, http://www.energymonitor.com

[3] X. Jiang, S. Dawson, P. Dutta, and D. Culler, Design and implementation of high-fidelity ac metering network, Proc. of IEEE Int. Conf. on Information Processing and Sensor Networks, California, USA, 2009.

[4] S. Keshav and C. Rosenberg, How internet concepts and technologies can help green and smarten the electric grid, Green Networking 2010, New Delhi, India, 2010.

[5] Y. Kim, T. Schmid, Z. M. Charbiwala,, and M. B. Srivastava Smith, Design and implementation of a fine grained power monitoring system for homes, Networked \& Embedded System Lab., 2009.

[6] Shum-Yu Chan and Jen-Han Teng H. Miller, Advance remote control infrastructure for intelligent HEMS, Proc. of Int. Conf. on Infomration and Electronics Engg., Singapore, 2011.

[7] D. D. Vyas and H. N. Pandya,Advance metering infrastructure and DLMS/COSEM standards for smart grid - A review, Int. Journal of Engg. Research and Tech., Vol. 1, No. 2, Nov. 2012.

[8] The home area network architectural considerations for rapid innovation, Penn Energy, 2009, http://www.pennenergy.com.

[9] CS5460A-Single phase bi-directional power energy IC Data Manual, Cirrus Logic.

[10] AVR ATmega32 Microcontroller Data Manual, Atmel Corporation.

[11] AN227, Calibrating the CS5460A, Cirrus Logic.

[12] External commands for AUBTM-20 Bluetooth Module, Austar Technologies.

[13] Bluetooth EDR: Wireless Evolution, Application note, Agilent Technologies, 2006.

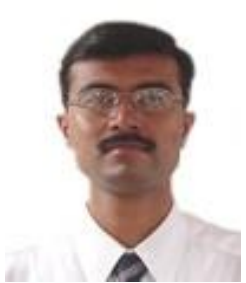

Divyang D. Vyas has obtained his B.E. in Instrumentation and Control Engineering from L. D. College of Engineering, Ahmedabad, in 1999 and the M.Tech. in Electronic Systems from Indian Institute of Technology, Bombay, in 2005. He is presently pursuing his Ph.D in Electronics and Communication Engineering at Saurashtra University, Rajkot. He has a teaching experience of more than 12 years and is presently working as Associate Professor and Head of Electronics and Communication Department at Darshan Institute of Engineering and Technology, Rajkot. His areas of interest are electronic circuits, signal processing and embedded systems. He is life member of ISTE and IETE.

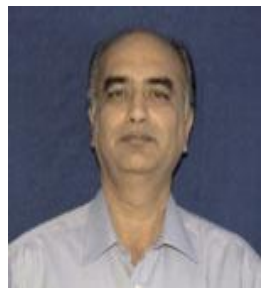

Dr. H. N. Pandya is presently serving as Professor and Head of Department of Electronics, Saurashtra University, Rajkot. He has a long teaching experience of more than 20 years and has successfully guided six Ph.D. thesis and number of Bachelors and Masters dissertations. He has published and presented number of research papers in Journals/Conference of repute and has authored three books. He has completed three minor research projects funded by UGC and has been awarded research fellowship from agencies like UGC, CISR etc. His areas of interest are electronic circuits, microprocessor, microcontrollers, embedded systems and ferrite materials. 